\title{
Odri: Oncology Database of Rims, Ranchi
}

\author{
Tannu Kumari ${ }^{1}$, Anupa Prasad ${ }^{2}$, Anup Kumar ${ }^{3}$, Ashish Ranjan Shukla ${ }^{4 *}$, \\ Kumar Satyam ${ }^{5}$ \\ 1, 4, 5 (Biomedical Informatics Centre, Department Of Biochemistry, RIMS, Ranchi, India) \\ ${ }^{2}$ (Department Of Biochemistry, RIMS, Ranchi, India) \\ ${ }^{3}$ (Department Of Radiotherapy, RIMS, Ranchi, India)
}

\begin{abstract}
Cancer is a group of diseases involving abnormal cell growth with the potential to invade or spread to other parts of the body. On one hand India had started national programs since 1975, and till date majority of the metropolitan cities have cancer registries but there is still lack of such repositories in rural and neglected areas. The department of oncology is superspeciality centre of cancer in the state. The oncology database of RIMS is developed at Biomedical Informatics Centre. ODRi is the only database in the centre and state that collects information on approximately all new cancer diagnosed in the state or referred from any other higher centres. The data are stored in a systemic manner where the user can search, edit and download the data. The database currently contains approximately 500 cancer data of all cancers reported from and across the state. The database currently holds data from 2014 to 2017. ODRi is freely accessible and in future will incorporate data from other cancer institute.
\end{abstract}

Keywords: Cancer, Database, Oncology

\section{Introduction}

Cancer is a group of diseases involving abnormal cell growth with the potential to invade or spread to other parts of the body [1, 2]. It has now become a common and serious health problem. The major risk factors include smoking, alcohol, obesity, poor diet etc. The repository for cancer is not new in the country. India has had a national program for cancer since 1975, where the emphasis was on equipping premier cancer institutions, and by 1984-1985, shifted to primary prevention and early detection of cancer cases and, by 1990-1991, to the district cancer control program. As of 2008, creation/recognition of new regional cancer centers, strengthening of existing regional cancer centers, development of oncology wings in medical college hospitals, the district cancer control program, and the decentralized NGO scheme were the priorities of the program [3]. In 2010, cancer control became a part of a more comprehensive, larger program on noncommunicable diseases called National Programme for Prevention and Control of Cancer, Diabetes, Cardiovascular Disease and Stroke (NPCDCS) where the common risk factors are addressed in an integrated manner [4, 5]. However in the absence of a nationwide screening program, there are disparities in screening, treatment, and also survival. Despite of the ample effort only registries or databases are built only in urban parts of the country and very few in rural areas. Majority of the rural area is still lacking behind. However the cases of cancer are growing day by day in the rural parts as well. In this scenario there is an urgent need to develop a registry that will emphasize on systematic collection, storage, analysis, interpretation and reporting of data on subjects with cancer. The main purpose of the database is to contribute to patient care by providing readily accessible information on the patients with cancer over the conventional file system. The data will be used for administrative purposes and for reviewing clinical performances.

\subsection{Data Collection}

\section{Materials And Methods}

The data is collected from the oncology department where in each of the patients has a specific registration number being issued by the hospital. Other demographic details such as name, sex date of birth/age, address, religion, phone no (if provided) are collected. The clinical information collected are the cancer type, stage, BMI, BP and the various blood parameters. 


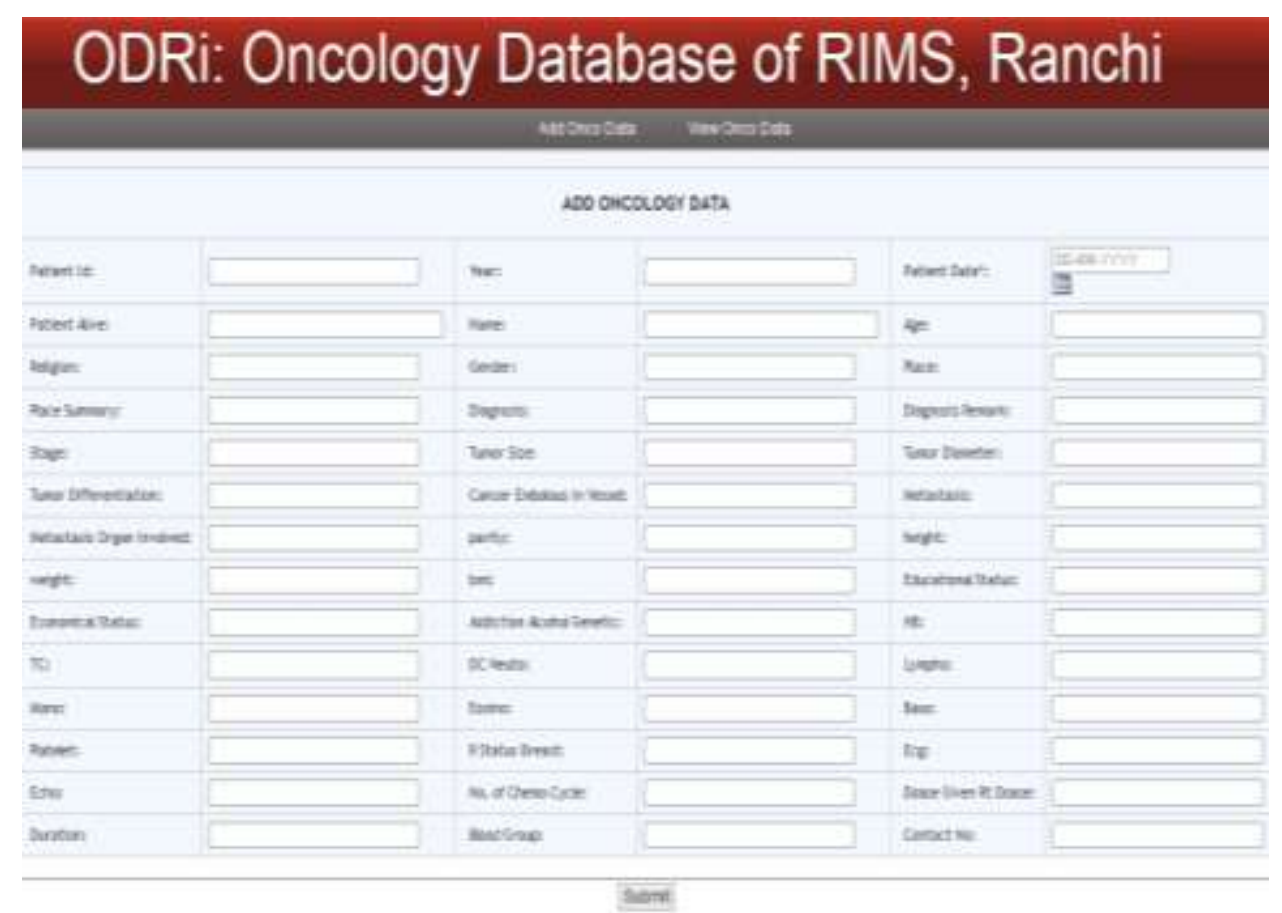

Figure 1: Format for data input

\subsection{Database Design}

The database is designed built on Apache HTTP Server 2.2.17. The database is created using MySQL Server 5.5.8 and the web interfaces are designed using CakePHP 1.3, PHP 5.3.5, HTML, CSS, JavaScript.

\subsection{Data Input}

The data can be added and edited by means of form. Once new data is added it upon pressing submit it will be directly saved into the database. Figure 1 shows the snapshot of the data input.

\subsection{Data Visualization}

The details of electronic data of cancer patients in can be viewed in various ways like:

a. View details by entering patient ID:

It will give the list of patients matching with the unique registration ID.

b. View details by entering patient name:

It will give the list of patients with matching or similar patient name

c. View details of patient by selecting the type of cancer:

It will give the list of patients those who have the selected type of cancer disease.

d. View details of patient by selecting the year:

It will give the list of patients enrolled in RIMS by selected year

\section{ODRi: Oncology Database of RIMS, Ranchi

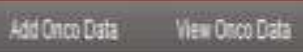

O Search By Name
O Search By Patient Id
O Search By Type of Cancer
O Search By Year
O View Total Data / Download Data
in Excel(.xls) Format
Suamit

Figure 2: Format for data retrieval /search 


\subsection{Data download}

One of the most important feature of the database is that the entered data can be downloaded by the end user in form of excel (.xlsx) and can be used for research or academic purpose.

\section{ODRi: Oncology Database of RIMS, Ranchi}

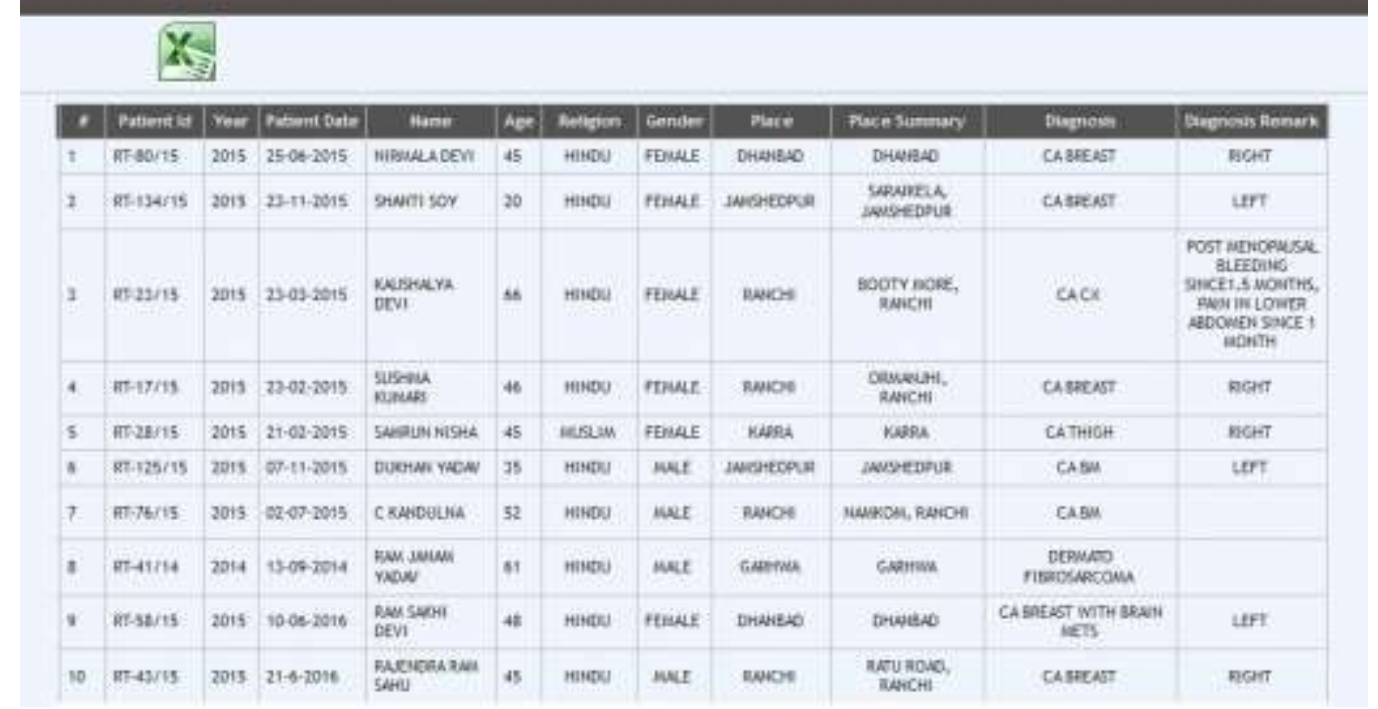

Figure 3: Detailed data view

\section{Results}

There are very few online databases catering to cancer data in hospitals, thus limiting the data to be used in research and academics. ODRi provides an excellent interface to clinicians and researchers to use the well-integrated information available in it. ODRi would be updated annually. The pie chart shows the distribution of cancer patient year wise.

\section{Percentage of Cancer Patients}

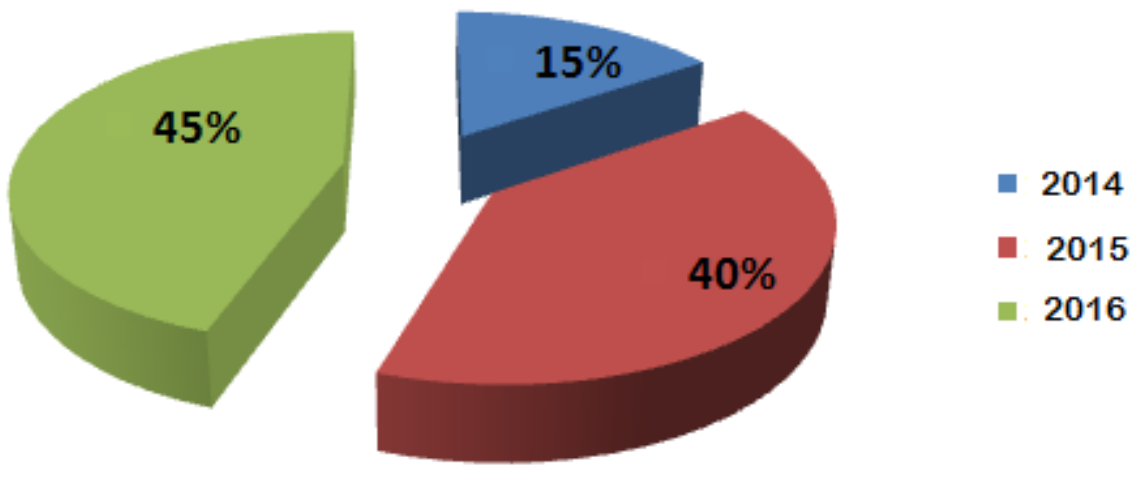

\section{Conclusion}

The ODRi is the first and only existing oncology database providing multiple opportunities for various research studies. The data from the department will be continuously updated with the combined effort of both the doctors and the scientists to improve the accuracy, completeness and quality of the hospital's cancer registry data, and thus the quality of the data reported to the ODRi 


\section{References}

[1]. "Cancer Fact sheet N²97". World Health Organization. February 2014. Retrieved 10 June 2014

[2]. $\quad$ "Defining Cancer". National Cancer Institute. Retrieved 10 June 2014

[3]. National Cancer Control Programme. Available from: http://www.mohfw.nic.in/showfile.php?lid=324. Accessed July 16, 2014.

[4]. National Programme for Prevention and Control of Cancer, Diabetes, Cardiovascular Disease and Stroke. Available from:http://www.nrhmhp.gov.in/sites/default/files/files/NCD_Guidelines.pdf

[5]. Sreedevi A, Javed R, Dinesh A. Epidemiology of cervical cancer with special focus on India. International journal of women's health. 2015;7:405. 